\title{
Distribution des vitesses moyennes pour l'écoulement turbulent dans les conduites cylindriques
}

\author{
Distribution of mean velocities \\ with turbulent flow in cylindrical pipes
}

PAR R. LEGENDRE

INGENIETh EN CHEY DL GLNIE MARITIME

Cne nowelle relation afferentielle entre la tarbulence el la force moyente de piscosité, inspirée des résultats de Reichanor, sert de base à rintégralion de la loi de disfribution des vilesses dans tonte la section, ainsi qu'au calcul du débit et du coefficient de frottement. L'ensemble des résultals relatifs à l'eoulement turbulent dans les conduites rylindriques est ainsi ratlache ì une loi simple qui, sans difficalté d'intégration insurmontable, définit linfluence du nombre de Reynolds ponr la totalité de la courbe de distribution des vitesses, sanf pentthe dans le domaine de transition.

\begin{abstract}
A new differential relation, inspired by RexcHARD's results, between turbulence and the mean force of viscosity, serves as a basis for integration of the velocity distribution law throughout the section, as well as for calculation of the discharge and of the friction roefficient. All the results relating to turbulent flow in cylindrical pipes are thus linked to at simple law which, without involving great difficullies of integration, defines the influence of the Reynold's namber for the entire velocity distribution curve, except perhaps in the transition field.
\end{abstract}

\section{INTRODUCTION}

Par une élude antérieure (1), nous avons tenté de représenter l'écoulement turbulent dans le

(1) Note sur la distribution des vitesses moyennes pour l'écotement turbulent dans les conduites cylinariques. La llouille Blanche, $\mathrm{n}^{*} \mathrm{~A}, 1948$ (Mém. ef Trav. S.II.F., $\mathrm{n}^{\circ} 1,1948$ ).

(2) J. Nkr habse. -.- Gesetzmässigkeiten des turbulente strömungen in glatten Bohren. Forschuagscheft, 356 , 1932.

(3) Film turbulent dans les conduites. La Houille Bimehe, mat 1950 .

(4) H. Reichanor. - Der Einfluss der wandnahen Strönung auf der turbulente Wärmeäbergang. Mitteitungen ais dem Max Planck Instifut, 1950.

(a) H. Buchandr. -... Vollstandige Darstellung der turbulenten Geschwindigkeits Vertejlung in ghtten Leitungen. Z.A.M.M., juillet 1051 . noyau central d'une conduite cylindrique à partir des résultats anciens de Ninunadse (2).

Nous avons, d'autre part, recherché une relation déterminant la turbulence dans le film au voisinage de la paroi (3) et assurant un raccordement continu de la loi de distribution des vitesses dans le noyau à la loi de distribution des vilesses dans le film.

Simultanément, Reichand publiait (4-5) de nouveaux résultats sur la distribution des vitesses et présentait une interpolation empirique très voisine do celle que nous avions proposée.

L'objet de la présente étude est une retouche de la relation délerminant la turbulence, faite en vue d'une extrapolation dans le noyau central 
avec représentation correcte des résultats de RIICHARDT.

\section{LOI REDUITE DU FROTTEMENT}

La loi réduite de frottement peut être représentée sous la forme simple :

$$
y=g+h
$$

où :

- $y$ est le rapport du rayon local au rayon $\mathrm{R}$ de la conduite;

- $g$ est le rapport de la pente locale de la courbe de distribution des vitesses moyennes $d u / d y$ à la pente de cette courbe à la paroi $d u / d y_{p}$

- $h$ est le rapport $u v / u_{*}{ }^{2}$ de la valeur moyenne du produit des composantes axiale et radiale de la vilesse au carré de la vitesse de frottement $u_{*}$ :

$$
u_{*}{ }^{2}=\tau / \rho=-\frac{\mathrm{R}}{2 \rho} \frac{d p}{d x}
$$

où $\tau$ est la tension de frottement à la paroi, $\rho$ la masse spécifique et $d p / d x$ le gradient de pression.

Il doit être noté que, la turbulence étant négligeable au voisinage immédiat de la paroi, la pente de la courbe de distribution des vitesses est déterminée par la viscosité seule.

$$
\tau=-\mu \cdot \frac{1}{\mathrm{R}} \frac{d u}{d y_{p}}
$$

où $\mu=\rho$ v est la viscosité absolue et $\nu$ la viscosité cinématique :

$$
-\frac{1}{u_{*}} \cdot \frac{d u}{d y_{1}}=\frac{u_{*} \mathrm{R}}{\nu}=\frac{1}{2 \varepsilon}
$$

et $\varepsilon$ est l'inverse du nombre de Reynolds formé avec la vitesse de frottement.

\section{RELATION DETERMINANT LA TURBULENCE}

Pour notre étude antérieure du film, nous avions introduit une relation complémentaire :

$$
\frac{d}{d y}\left\lceil\frac{h^{1 / 3}}{g}\right]=-\frac{k h}{2 \varepsilon}
$$

où $k$ est la constante de Karman.

Nous avions signalé que cette relation ne peut pas être extrapolée dans le noyau.
La présente étude est fondée sur la relation :

$$
\frac{d}{d y}\left[\frac{h}{g}\left(\frac{h}{y}\right)^{1 / 3}\right]=-\frac{k h}{2 \varepsilon} \frac{4 y^{2}-1}{3}
$$

L'introduction de $y$ dans le premier membre a essentiellement pour objet de conserver un sens a la relation pour les valeurs négatives de $y$. Elle n'altère pratiquement pas la loi des vitesses dans le film, où $y$ est très voisin de 1 , et simplifie l'extrapolation dans le noyau, où $h / l$ est très voisin de 1 .

L'introduction dans le second nombre de la fonction $\frac{4 y^{2}-1}{3}$, qui est égale à 1 à la paroi, est destinée à la représentation des résultats de REICHARDT.

\section{PENTE DE LA COURBE DE DISTRIBUTION DES VITESSES}

La relation ci-dessus peut ètre facilement intégrée et définit la pente de la courhe de distribution des vitesses en fonction du rayon réduit $y$. Posons en effet :

$$
h=y-g=y \mathrm{~S}
$$

Alors la relation prend la forme d'une écuation différentielle à variables séparées :

$$
\frac{4-\mathrm{S}^{:}}{\left(1-\mathrm{S}^{\prime}\right)^{2}} d \mathrm{~S}=-\frac{k}{2 \varepsilon} \frac{4 y^{2}-1}{3} \eta d y
$$

Pour les conditions limites a la paroi $s=0$ et $y=1$, elle s'intigre immédiatement par :

$$
\begin{gathered}
I(S)=\frac{S}{1-S^{3}}+\frac{1}{2} \ln \frac{1+S+S^{2}}{(1-S)^{2}} \\
+\sqrt{3} \operatorname{Arctg} \frac{\sqrt{3} S}{2+S}=\frac{k}{12 \approx}\left(1-y^{2}\right)\left(1+2 y^{2}\right)
\end{gathered}
$$

Lorsque $y$ décroil de 1 à 0 , la valeur commune des deux membres croît de 0 à $3 / 32 \mathrm{k} / \varepsilon$ pour $y=1 / 2$, puis décroil jusqu'à $k / 12$ \& pour $y=0$. Parallèlement, $S$ croit de 0 à une valeur $S_{1}(k / \varepsilon)$ pour $y=1 / 2$, puis décroît jusqu'à une valeur $\mathrm{S}_{0}(k / \varepsilon)$ pour $y=0$. Ces valeurs sont déterminées par :

$$
\mathrm{I}\left(\mathrm{S}_{1}\right)=\frac{3}{32} \frac{k}{\varepsilon} \quad \mathrm{I}\left(\mathrm{S}_{0}\right)=\frac{1}{12} \frac{k}{\varepsilon}
$$

La variable $S$ reste toujours inférieure a 1 , mais s'en approche d'autant plus que e est plus petit, c'est-à-dire que le nombre de Rwowoms est plus grand.

La variation de $\mathrm{S}$ avec $y$ étant ainsi détermi- 
née, la pente réduite de la courbe de distribution de vitesse $1 / u_{*} d u / d y$ est définie par :

$\frac{1}{u_{*}} d u / d y=-\frac{\eta}{u_{*}} d u / d \eta_{p}=\frac{g}{2 \varepsilon}=\frac{y\left(1-\mathrm{S}^{3}\right)}{2 \mathrm{\varepsilon}}$

\section{DISTRIBUTION DES VITESSES DÉBIT ET COEFFICIENT DE FROTTEMENT}

bes intégrations supplémentaires sont nécessaires pour le calcul de la vitesse locale, du débit el du coefticient de frottement.

Ial distribution des vitesses est définie par:

$$
\frac{n}{n_{*}}=\frac{1}{2 \varepsilon} \int_{y}^{+1}\left(1-S^{*}\right) y d y
$$

Lat vitesse déficitaire, difrérence de la vitesse maximum $u_{m}$ sur laxe ef de la vilesse beale $n$, est délerminée par :

$$
\frac{u_{m}-u}{u_{*}}=\frac{1}{2 \varepsilon} \int_{0}^{y}\left(1-\mathrm{S}^{*}\right) y d y
$$

Ie débit est te produit de la seclion par la masse specifique et par la vitesse moyenne r' dans la section difinie par :

$$
\frac{V}{u_{*}}=\frac{1}{2 *} \int_{0}^{y}+1 \quad S * ! y^{*} d y
$$

Enfin, le coefficient de frollement $\mathfrak{c}^{3}$ est domné, sans inlégration nouvelle, par :

$$
c_{1}=2 \frac{u_{*}^{*}}{r^{\prime \prime 2}}
$$

II n'est fonction, comme $V / u_{*}$, que de $s, c^{\prime} e s t-$ a-dire du nombre de Rexsolos.

\section{SOLUTION EXACTE}

Le rayon róduit peul être exprimé explicifement en fonction du parametre $s$ et la solulion cxacte peut ètre calculée numériquement pir les formules:

$$
\begin{aligned}
& 4 y_{3}^{*} \quad 1=11-\frac{32}{3} \frac{3}{k} \mathrm{I}(\mathrm{S})
\end{aligned}
$$

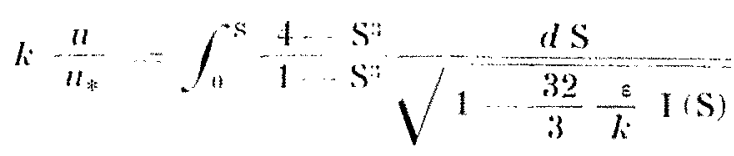

$$
\begin{aligned}
& \text { \& } \frac{V^{r}}{n_{*}}=\int_{n}^{+K_{*}} \frac{4-S^{3}}{1 \cdots S^{3}}\left[1+\frac{3}{\sqrt{1-\frac{32}{3} \frac{\varepsilon}{k} \mathrm{I}(\mathrm{S})}} \mid \frac{d \mathrm{~S}}{4}\right.
\end{aligned}
$$

Si la détermination négative du radical est retenue pour le calcul de $y<0,5$, il convient de proceder aux intégralions d'abord de 0 a $S_{1}$ pour la détermination positive du radical, puis de $S_{\text {, }}$ a $S$ ou à $S_{0}$ pour la valeur négative du radieal.

La formule d'intégration montre que pour !/ $<0,5:$

$$
u\left(y^{2}\right)=2 n(1 / 4) \cdots n\left(1 / 2-\cdot y^{2}\right)
$$

La solution exacte peut présenter de l'intérèt pour l'étude précise des faibles nombres de REYvouss dans le domaine de raccordement du régime turbulent au régime laminaire. Elle ne sera pas exploitée par la suite.

\section{DISTRIBUTION APPROXIMATIVE DES VITESSES}

Mettant en évidence Pallure de variation de la vitesse au voisinage de la paroi, on peut cerire:

$$
x \cdot \frac{n}{n_{*}}=J(\mathrm{~S})+\mathrm{A}\left(\eta^{2}, \varepsilon\right)
$$

ave :

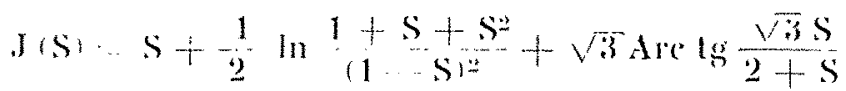

En dérivant el tenant comple de la relation entre $y /$ et $s$, il est possible d'obtenir :

$$
\frac{d \mathrm{~A}}{d y^{2}}=4 \frac{1-\mathrm{S}^{3}}{1+2 y^{2}} \mathrm{I}(\mathrm{S})
$$

Le produil (1 $\ldots-S \%$ I S S est nul pour $S=0$, mais tend vers 1 lorsque $S$ s'approche de 1 et, pour une correction, il peut suffire de le confondre avec $1(*)$. Alors:

$$
A \sim 2 \ln \frac{3}{1+2 y^{2}}
$$

En définilive, la distribution des vitesses est définie paramétriquement, en premiere approximation, par :

$$
\begin{aligned}
& \frac{+y^{2}-1}{3}=\sqrt{1 \cdots \frac{32}{3} \frac{\varepsilon}{k} \mathrm{I}(\mathrm{S})} \\
& k \frac{u}{u_{*}} \sim J(\mathrm{~S})+2 \mathrm{LI} \frac{3}{1+2 y^{2}}
\end{aligned}
$$

(*) Cone integration par parlies pourail fournir une approximation plus seréc :

$$
A-2(1-\mathrm{s} 3)(x) \ln \frac{3}{1-2 ! r^{2}}+\ldots
$$


En particulier, la vitesse maximum sur l'axc $u_{m b}$ peul etre calculée par :

$$
k \frac{u_{m}}{u_{*}}-\mathrm{J}\left(S_{0}\right)+2 \ln 3
$$

Lat valcur correspondante de $\varepsilon$, c'est-à-dire du nombre de Rirvorns, est donnée par :

$$
\varepsilon=\frac{k}{12 \mathrm{I}\left(\mathrm{S}_{0}\right)}
$$

\section{CALCUL DE LA DISTRIBUTION DES VITESSES}

Les résultats du calcul sont représentés sur la figure 1.

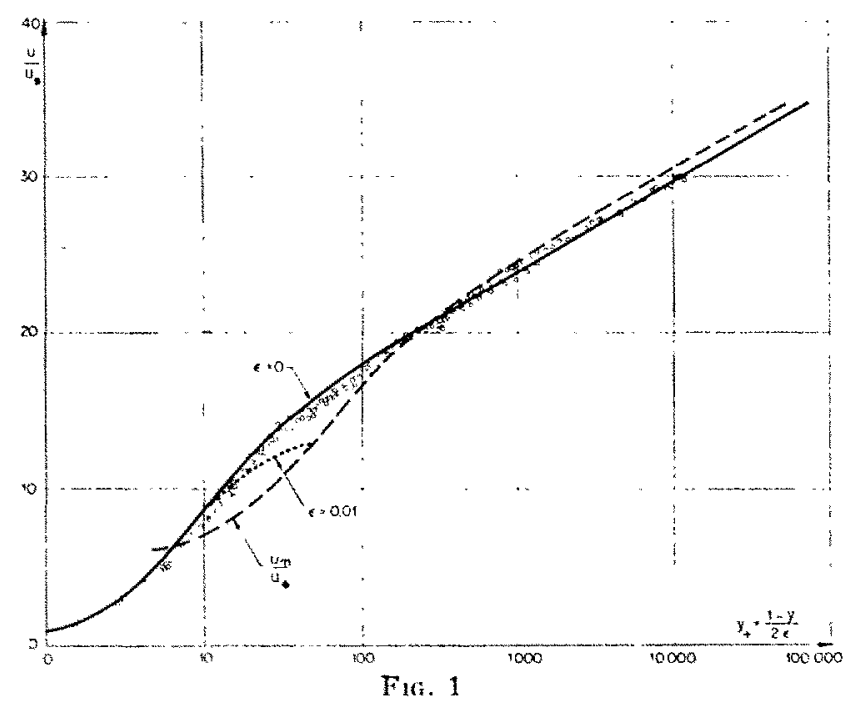

La courbe en trait plein correspond a la limite pour $\varepsilon=0$ de la distribution des vilesses $u / u_{*}$ porlée en fonction de $I_{+}=(1 \ldots y) / 2 \varepsilon$ sur une échelle logarithmique.

La courbe en trait discontinu correspond a la vitesse maximum réduite $u_{m} / u$ en fonction de $y_{+}=\frac{1}{2 \varepsilon}$ pour $y=0$. Elle cesse detre significative pour les petites valeurs de $y$, en raison des approximations faites.

Lal courbe en trait ponclue represente la distribution des vitesses pour $=0,01$. Elle n'est pas connue avec précision, puisque les approximations supposent a tres petit, mais donne une image de l'influence du nombre de Rexolds.

Les courbes correspondanl a des valeurs de a plus laibles, du domaine des nombres de RryNoLds usuels, n'ont pas été tracies car elles sont pratiquement confondues avee la courbe limite pour $\varepsilon=0$ lorsque $y_{+}$est modéré et s'élèvent ensuite légèrement au-dessus de celle courbe pour franchir même la courbe des vilesses maxima avant dy aboutir.
Les points expérimentaux de Nuresdse of de Riachard ont été reportés sur la figure sans indication du nombre de Rryvol.ps ou de la valeur de a car ils correspondent lous à des nombres de Rrivondos assez devés et il est dija salisfaisant de constater qu'ils sont voisins de la courbe limite et plutôt au-dessous dans la région $10<y_{+}<100$ ou les courbes théoriques nontrent précisement que la vitesse doit diminuer pour une valeur donne de $y_{+}$lorsque z eroit.

Lat representation des résultats expérimentaux dins le domaine des grandes valeurs de $y$. est un peu moins satisfaisante.

\section{COEFFICIENT DE FROTTEMENT APPROXIMATIF}

I.e procédi d'approximation, ulilisé pour lo caleul de la distribution des vitesses, peul aussi itre exploite pour la determinalion de fa vilesse moyenne dans la section, mais lerreur introduite dans chacun des ealeuls ne garantit pas lem parfaite correspondance.

Lat vitesse moyenne est lat limile pour $! /=0$ de $\mathrm{V}(y)$ :

$$
\frac{V(y)}{n_{*}} \quad \therefore \frac{1}{2 \varepsilon} \int_{y}^{1}\left(1-\mathrm{S}^{\prime}\right) y^{*} \cdot d !
$$

Posons :

$$
k \frac{V(y)}{u_{*}} \quad \mathrm{~J}(S)+B\left(y^{2}, z\right)
$$

La derivalion el lélimination parlielle de y doune:

$$
\begin{gathered}
\frac{d B}{d y^{2}}=-\frac{1}{1+\frac{S}{2} y^{2}}+(S)=\frac{1}{4} \frac{d A}{d y^{2}} \\
B=\frac{A}{4}-\frac{1}{2} \ln 1-\frac{3}{1-2} y^{3}
\end{gathered}
$$

el, en passanl à la limite:

$$
\text { li. } \frac{V}{n_{*}} \sim J\left(S_{n 1}\right)+\frac{1}{2} \ln : 3
$$

ou encore. en rapplochan de la loi de vilesse maxima of an atoptant $k=0,4$ :

$$
u_{m} \frac{v}{u_{*}}-\frac{3}{2 k} \ln 3=\frac{15}{4} \ln 3-4,12
$$

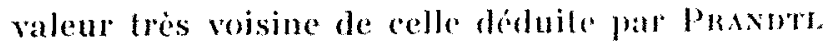
des résultals expérimentanx. soil 4,07 .

Il est alors facile de calculer le coeffecient de 
froltement $\vec{C}$, et le nombre de Rryoubs a correspondant en fonction du paramètre $S_{0}$ :

$$
\begin{gathered}
k \sqrt{\frac{2}{\mathrm{C}_{*}}}=J\left(\mathrm{~S}_{0}\right)+\frac{1}{2} \ln 3 \\
R=\frac{2 \mathrm{VR}}{\mathrm{V}}=\frac{1}{\varepsilon} \frac{\mathrm{V}}{u_{*}}=\frac{12 \mathrm{I}\left(\mathrm{S}_{0}\right)}{k} \sqrt{\frac{2}{\mathrm{c}_{1}}}
\end{gathered}
$$

Les résutats du calcul sont représentés sur la figure 2, où ils sont comparés avec les résultals expérimentaux.

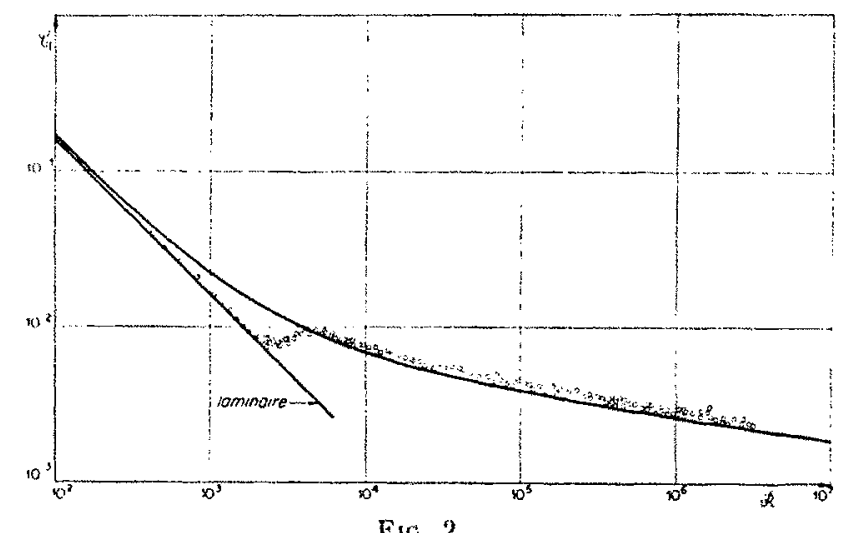

Dans le domaine franchement turbulent, le coefficient de frottement calculé est un peu trop faible. Ceci doit être rapproché de la valeur trop élevée de la vitesse maxima, déjá mise en évidence sur la figure 1 , et qui entrine la vitesse moyenne. Il ne serait possible de remédier à cette imperfection qu'en retouchant quelque peu la loi de couche-limite ou la loi d'extrapolation vers le noyau de Reichandt. Nous avions antérieurement étudié la relation :

$$
I(S)=\frac{k}{8_{\varepsilon}}\left(1-y^{4}\right)
$$

qui correspond à la relation complémentaire simple :

$$
\frac{d}{d y}\left[\frac{h}{g}\left(\frac{h}{y}\right)^{1 / 3}\right]=-\frac{k}{2 \varepsilon} h y^{2}
$$

ef conduit a une valeur plus satisfaisante de la vitesse maxima, de la vitesse moyenne et du cocfficient de frottement, mais s'accorde moins bien avec la distribution des vitesses dans le noyau telle qu'elle est présentéc par Reichandr.

Dans le domaine de transition, les résultats expérimentaux montrent un raccordement du régime turbulent au régime laminaire beaucoup moins progressif que celui qu'indique le calcul. Il est vraisemblable que ceci subsisterait s'il était renoncé aux facilités des approximations qui sont plus spécialement hasardées dans ce domaine.

\section{CONCLUSION}

Il semble possible de représenter l'ensemble des résultats expérimentaux relatifs à l'écoulement dans les conduites cylindriques en partant d'une loi élémentaire assez simple qui, sans difficulté d'integration insurmontable, définit l'influence du nombre de Rrvolds pour la totalité de la courbe de distribution des vitesses, sauf peutètre dans le domaine de transition.
Il est vraisemblablement possible d'améliorer cncore la représentation en retouchant quelque pou la loi écémentaire. Celle-ci n'a pourtant pas de signification physique profonde car il est vraisemblable que la turbulence satisfait à une équation integrale dont les équations différentielles, progressivement compliques depuis Von KARman, ne donnent qu'une image approchée.

\section{DISCUSSION}

(President : M. BARnuLON)

II. Daxist slgnale que M. Bresser a fat récemment une elude analogue basé sur des hypotheses simplifiées ef définissant théoriquement la harpe et la courbe de Nikuradse, mais s'écartant de la droite de Blasius pour redescendre vers le bas après le genou. Cette theoric. expliquerait certains résultats d'expériences, notamment colles de Comoner, et tendrait a résoudre le problème de la stabiliti de lecoulement laminaire.

M. Legexme remarque que le genou est beaucoup moins accentué si on lemplace l'echelle des coefficients do poltement par cello des résistances: or, dans la stabilite, c'est la résistance, produit du cocfficient de frottement par le camé de la vilesse qui compte. M. Legendr: pensait qu'il pouvait exister pour des vitesses croissantes un faux équilibre laminaire prolongé et qu'en revanche, si on diminuait la vitesse, on pouval se raccorder d'une façon continue í la courbc, par suite du retour de la turbulence.

M. le Président remercie M. Legwnone et propose de demander à M. Biśsel de présenter son étude à une pro-
chaine réunion. 